\title{
Should patients with lymphangioleiomyomatosis undergo screening for meningioma?
}

\section{To the Editors:}

Pulmonary lymphangioleiomyomatosis (LAM) may be sporadic or associated with tuberous sclerosis complex (TSC). Recently, routine screening for brain abnormalities associated with TSC demonstrated the presence of meningioma in $3.2 \%$ of patients with LAM (with or without TSC), a prevalence exceeding that expected in the general population [1], thus suggesting that meningiomas may be associated with LAM or its treatment. Indeed, most patients diagnosed with meningioma (seven out of eight) had received hormone replacement therapy and/or progesterone. Hitherto reported cases of meningiomas associated with LAM were mostly asymptomatic [1], with ensuing controversy about the usefulness of systematic brain imaging in patients with LAM [2]. We report the occurrence of symptomatic meningiomas requiring surgery in a patient who had received progesterone therapy for 17 yrs for pulmonary LAM.

A female born in 1946 was diagnosed in 1985 with pulmonary LAM, with dyspnoea on exertion, occasional chyloptysis, and characteristic diffuse cystic lesions on chest computed tomography (CT). Spirometry was normal, carbon dioxide diffusing capacity of the lung was $54 \%$ predicted, and resting arterial oxygen tension was $9.3 \mathrm{kPa}$. The patient had no criteria of TSC. Brain CT was not performed. The diagnosis of LAM was established by open lung biopsy. Immunohistochemical studies showed expression of progesterone (but not oestrogen) receptors by LAM cells. Serum levels of oestrogens and progesterone were normal. Therapy with progestative drugs was started, with i.m. medroxyprogesterone $500 \mathrm{mg}$ monthly for $1 \mathrm{yr}$, then oral nomegestrol $10 \mathrm{mg}$ q.d. Follow-up showed slow impairment of pulmonary function. Oxygen therapy was started in October 2003.

The patient was admitted in December 2003 because of behavioural changes, gait disturbances, and bladder and bowel incontinence. Examination revealed major astasia, symmetric brisk reflexes, apragmatism, and decrease in attention and verbal fluency. Cerebral CT showed a right fronto-temporal tumour, $7 \mathrm{~cm}$ in diameter (fig. 1), with peritumoural oedema, together with a smaller parasagittal lesion, both suggestive of meningiomas. Surgical resection of the fronto-temporal mass resulted in rapid and complete recovery of the neurological and cognitive status. Histological examination of the tumour showed meningothelial meningioma, with expression of progesterone (but not oestrogen) receptors by tumour cells.

Since LAM occurs mainly in females of childbearing age, a role of oestrogens in the progression of pulmonary LAM has been suspected. Progesterone treatment is therefore commonly prescribed, despite lack of evidence of beneficial effect. Both in vitro models and epidemiological studies suggest that female sex hormones may increase the risk of meningioma, consistent with a higher incidence among females than males [3], and the accelerated growth of meningioma during pregnancy. A large study recently found a relative risk of meningioma of 2.48 (95\% confidence interval 1.29-4.77) in pre-menopausal females who received hormone therapy [4]. A total of $50-80 \%$ of meningiomas express progesterone receptors

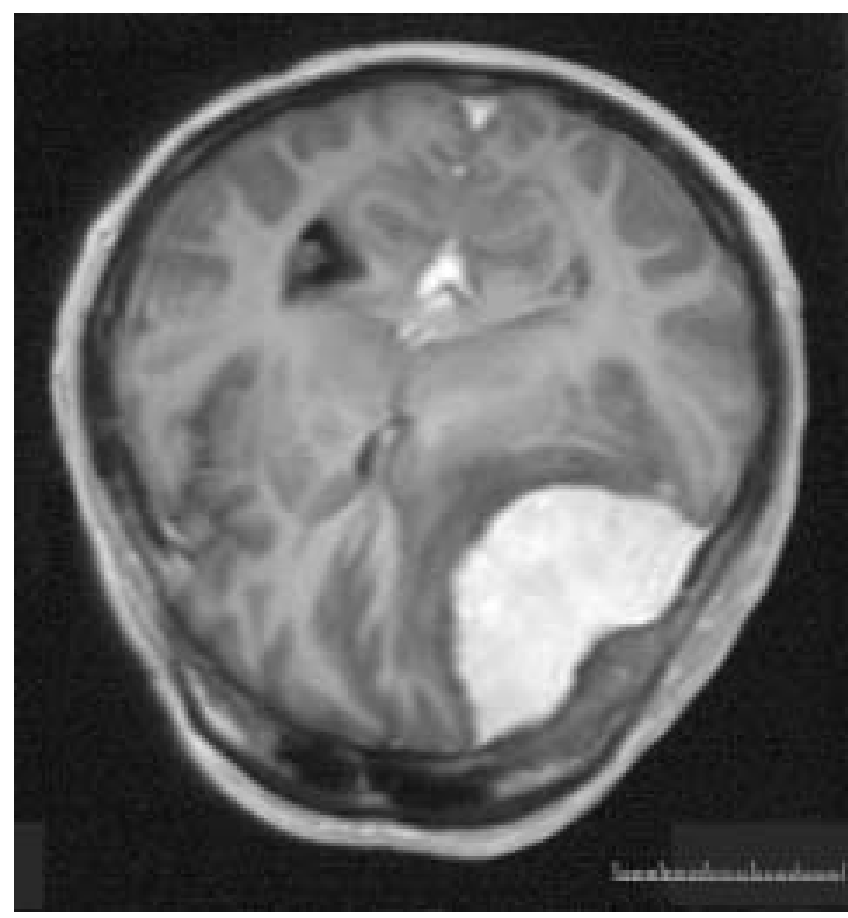

Fig. 1.-Axial gadolinium-enhanced T1-weighted magnetic resonance imaging showing a large right fronto-temporal meningioma, surrounded by oedema and significant mass effect on the brain. Scale bar $=5 \mathrm{~cm}$.

[5], with conflicting results regarding the influence of progesterone on the growth of meningioma cells in vitro.

Although the relationship between LAM and meningioma remains to be clarified, a link between the two disorders cannot be excluded. Amplification of S6 kinase (a protein involved in a critical pathway regulating cell growth and proliferation) has been reported in some meningioma cells, thus sharing biological similarities with LAM cells in which S6 kinase is activated [6, 7]. Somatic mutations of the TSC2 gene have been found in renal angiomyolipomas and lung LAM cells of patients with sporadic pulmonary LAM [8]; it remains to be determined whether similar mutations may be present in meningiomas.

As meningioma may be more than just an incidental finding in patients with lymphangioleiomyomatosis [2], we consider that lymphangioleiomyomatosis patients who are already on hormonal therapy or in whom such treatment is considered undergo brain imaging for screening of meningioma. Furthermore, we suggest that patients should be informed of the potential risk of a growth-enhancing effect of intracranial benign tumours before this treatment, with hitherto no proven benefit in lymphangioleiomyomatosis, is started.

\section{Cottin*, S. Vukusic ${ }^{\#}$, E. Jouanneau ${ }^{\top}$, R. Lazor ${ }^{+}$,} J-F. Cordier* 
* Service de Pneumologie, Centre des Maladies Orphelines Pulmonaires, Hôpital Cardiovasculaire et Pneumologique Louis Pradel, Université Claude Bernard and Unité Mixte de Recherche 754, "Service de Neurologie A, and "Service de Neurochirurgie A, Hôpital Pierre Wertheimer, Lyon, France. ${ }^{+}$Service de Pneumologie, Hôpitaux Universitaires de Genève, Geneva, Switzerland.

\section{References}

1. Moss J, DeCastro R, Patronas NJ, Taveira-DaSilva A. Meningiomas in lymphangioleiomyomatosis. JAMA 2001; 286: 1879-1881.

2. Franz DN. Meningiomas in women with lymphangioleiomyomatosis. JAMA 2002; 287: 1397-1398.

3. Longstreth WT Jr, Dennis LK, McGuire VM, Drangsholt
MT, Koepsell TD. Epidemiology of intracranial meningioma. Cancer 1993; 72: 639-648.

4. Jhawar BS, Fuchs CS, Colditz GA, Stampfer MJ. Sex steroid hormone exposures and risk for meningioma. $J$ Neurosurg 2003; 99: 848-853.

5. Hsu DW, Efird JT, Hedley-Whyte ET. Progesterone and estrogen receptors in meningiomas: prognostic considerations. J Neurosurg 1997; 86: 113-120.

6. Cai DX, James CD, Scheithauer BW, Couch FJ, Perry A. PS6K amplification characterizes a small subset of anaplastic meningiomas. Am J Clin Pathol 2001; 115: 213-218.

7. Krymskaya VP. Tumour suppressors hamartin and tuberin: intracellular signalling. Cell Signal 2003; 15: 729-739.

8. Carsillo T, Astrinidis A, Henske EP. Mutations in the tuberous sclerosis complex gene TSC2 are a cause of sporadic pulmonary lymphangioleiomyomatosis. Proc Natl Acad Sci USA 2000; 97: 6085-6090.

\section{Strategies to increase the lung donors' pool}

\section{To the Editors:}

In a recent issue of the European Respiratory Journal, DE PERRot et al. [1] suggested various strategies in order to increase the number of lung donors. Among these strategies, the extension of the lung donors' selection criteria was discussed. We would like to stress some points in a subtype of marginal donors. Most of the transplant coordination centres are reluctant to consider donors who present with brain death after a toxic exposure.

Data available from the organ procurement organisations are scarce. Cumulative data recovered from January 1988 to March 2004 by the US Organ Procurement and Transplantation Network revealed that, among the 10,981 lung donors recorded within the interval, the mechanism of death was drug intoxication in only 92 cases $(<1 \%)$. Similarly, the experience published by Eurotransplant (Austria, Belgium, Germany, Luxemburg and The Netherlands) is extremely limited. In 1996, a total of 168 lung transplantations were performed. The cause of death was mentioned as suicide for 15 donors $(9 \%)$. As suicide may have also included deaths secondary to gunshots, hanging or drowning, the overall percentage of intoxicated donors appears to be very low in this report.

In our centre (Dept of Intensive Care, Cliniques St-Luc, Université catholique de Louvain, Brussels, Belgium), from 293 organ donors, 864 organs were procured between January 1989 and December 1997. In contrast to the previous data, 21 $(7 \%)$ of our patients had developed brain death after acute poisoning [2]. A large number of intoxicants were used: cyanide, carbon monoxide, tricyclic antidepressants, barbiturates, paracetamol, insulin, and methanol. The outcome of the recipients of various organs was excellent. Bipulmonary transplantation was possible from a donor who died from methanol poisoning [3]. In the rejected cases, the lungs were not harvested for reasons that were independent from a toxic origin.

It is our experience that poisoned donors are often discarded a priori from organ donation, for fear of transferring the poisoning to the recipient with resultant organ dysfunction. However, the lung is seldom the main target organ of acute poisoning, with the notable exception of paraquat. Lung donation should be possible after acute overdose with the most frequent pharmaceutical drugs, including psychotropes.

Although there are data suggesting tricyclic antidepressants (TCA) accumulation in the lungs of a limited number of healthy volunteers, these data should not be extrapolated to transplant patients without caution [4]. There are data suggesting the feasibility of liver transplant in patients who died of TCA overdose and in whom TCA accumulation in the liver was demonstrated [5]. Accordingly, we believe that TCA overdose per se should not preclude a lung transplant.

Whereas fatalities after carbon monoxide exposure remain common, few numbers of lung transplantations have been reported [6]. It seems important to identify the source of carbon monoxide poisoning. Whilst various pulmonary lesions have been described following smoke inhalation, pure carbon monoxide poisoning is unlikely to be associated with chemical smoke-inhalation injury to the lung and lower airways [7].

Deaths in young donors are now frequently observed among illicit substance abusers. Successful lung transplantation has been performed from a donor who died after methylenedioxymethamphetamine ("ecstasy") exposure [8]. From a toxicological point of view, there is no reason to exclude ecstasy-intoxicated donors, provided that the commonly accepted criteria for organ donation are fulfilled.

In contrast, methadone has been reported to accumulate not only in the kidneys, spleen and liver, but also in the lungs. This should be particularly true in patients under maintenance therapy with multiple dosing. Acute lung injury is a rare but possible complication of acute methadone overdose [9].

The issue of organ donation after chronic use of inhaled drugs is still controversial. The pulmonary complications of inhaled cocaine are characterised by lesions of the alveolar wall and of the pulmonary microcirculation [10-12]. Shortterm exposure to cocaine may enhance the production of interleukin-8, a potent polymorphonuclear chemoattractant and neutrophil-activating factor, associated with both acute and chronic lung injury [12]. These data clearly preclude lung donation in these cases.

Regular marijuana smoking can induce alterations in the structure and function of alveolar macrophages, and these patients' lungs are unsuitable for transplantation [13]. This 